\title{
ESTRATÉGIAS PARA AVANÇOS NA PRÁTICA DO CUIDADO DOMICILIAR
}

\author{
Maria Ribeiro Lacerda ${ }^{1}$, Ingrid Meireles Gomes ${ }^{2}$, Luísa Canestraro Kalinowski ${ }^{3}$, Clélia Mozara Giacomozzi ${ }^{4}$
}

\begin{abstract}
RESUMO: Neste artigo apontam-se estratégias que operacionalizam avanços no cuidado domiciliar na Estratégia Saúde da Família (ESF), como resultado de um estudo prévio intitulado "A prática de cuidado domiciliar dos profissionais do Programa Saúde da Família". Utilizou-se a metodologia da pesquisa-ação com 27 profissionais de saúde de uma Unidade ESF. Foram elencadas 19 estratégias, agrupadas em 6 diferentes categorias temáticas, sendo elas: planejamento e organização do serviço; preparo profissional; condições que permeiam o cuidado domiciliar; revisão e fortalecimento dos saberes; melhor proveito da realidade; e discussões integradoras. As estratégias apresentadas revelam a necessidade de um envolvimento de todos os níveis políticos - participação popular, profissional e estatal - para a modificação da realidade de saúde do Brasil.
\end{abstract}

PALAVRAS-CHAVE: Estratégias; Programa saúde da família; Assistência domiciliar; Prática profissional.

\section{STRATEGIES FOR ADVANCES IN THE HOME CARE PRACTICE}

\begin{abstract}
In this article strategies are pointed out to operationalize advances in home care within the Family Health Strategy (FHS) as a result of a previous study entitled "The practice of home care of the Family Health Program professionals". We used the methodology of action research with 27 health professionals from an FHS Unit. 19 strategies were listed, grouped into six different themes: service planning and organization; professional preparation; the conditions that permeate the home care; review and strengthening of knowledge; better utilization of reality; and inclusive discussion. The strategies presented show the need for involvement of all political levels - popular, professional and government participation - to change the reality of health in Brazil.
\end{abstract}

KEYWORDS: Strategy; Family health program; Home care services; Professional practice.

\section{ESTRATEGIAS PARA AVANCES EN LA PRÁCTICA DEL CUIDADO DOMICILIAR}

RESUMEN: En este artículo, son indicadas estrategias que posibilitan avances en el cuidado domiciliar en la Estrategia Salud de la Familia (ESF), como resultado de un estudio previo intitulado "La práctica del cuidado domiciliar de los profesionales del Programa Salud de la Familia”. Se ha utilizado la metodología de investigación-acción con 27 profesionales de salud de una Unidad ESF. Fueron relacionadas 19 estrategias, agrupadas en 6 distintas categorías temáticas, siendo: planeamiento y organización del servicio; capacitación profesional; condiciones que permeian el cuidado domiciliar; revisión y profundización de los saberes; mejor provecho de la realidad; y discusiones integradoras. Las estrategias presentadas indican la necesidad de un envolvimiento de todos los nibeles políticos - participación popular, profesional y estatal - para el cambio de la realidad de salud de Brasil.

PALABRAS CLAVE: Estrategias; Programa salud de la familia; Asistencia domiciliar; Práctica profesional.

${ }^{1}$ Enfermeira. Doutor em Enfermagem. Professor Adjunto do Departamento de Enfermagem da Universidade Federal do ParanáUFPR. Coordenadora do Programa de Pós-Graduação em Enfermagem da UFPR. Coordenadora do Núcleo de Estudos, Pesquisa e Extensão em Cuidado Humano de Enfermagem-NEPECHE/UFPR.

${ }^{2}$ Enfermeira do Hospital Nossa Senhora da Luz. Membro do NEPECHE.

${ }^{3}$ Enfermeira. Mestranda do Programa de Pós-Graduação em Enfermagem na UFPR. Bolsista da Coordenação de Aperfeiçoamento de Pessoal de Nível Superior (CAPES). Membro do NEPECHE.

${ }^{4}$ Enfermeira do Hospital de Clínicas-HC/UFPR. Mestre em Enfermagem.

\author{
Autor correspondente: \\ Maria Ribeiro Lacerda \\ Universidade Federal do Paraná \\ R. Padre Anchieta, 198 - 80410-030. Curitiba-PR-Brasil \\ E-mail: lacerda@milenio.com.br
}

Recebido: 16/12/09

Aprovado: 01/09/10 


\section{INTRODUÇÃO}

No Brasil, discute-se amplamente sobre a necessidade de um modelo de atenção à saúde que contemple e atenda as diferentes necessidades e expectativas populacionais referentes às questões de saúde. Em busca de uma alternativa ao modelo biomédico de assistência, a Estratégia Saúde da Família (ESF) foi a principal proposta do Ministério da Saúde para a reorganização da Atenção Básica no território brasileiro ${ }^{(1)}$, por focalizar o núcleo familiar e caracterizar-se por ações preventivas.

A proposta da ESF representa uma nova perspectiva na política pública de saúde brasileira, que vai além da reabilitação ou recuperação de patologias. Nela, o indivíduo é compreendido como membro atuante no ambiente social, considerando-se as repercussões deste ambiente e reconhecendo esse indivíduo como co-participante no processo de saúde. Essa proposta tende a gerar uma melhoria progressiva na saúde da população e busca minimizar as desigualdades, de forma a se aproximar do ideal de equidade ${ }^{(2)}$.

A ESF capacita os profissionais para o conhecimento da realidade e para seu envolvimento e interação com a comunidade, sendo essencial o trabalho interprofissional para que a assistência atenda as reais necessidades de saúde ${ }^{(3)}$. Para tal, utiliza o cuidado domiciliar como um de seus recursos.

O cuidado domiciliar, também nomeado assistência ou atendimento domiciliar, representa um conjunto de ações desenvolvidas pelos profissionais de saúde no domicílio do usuário, abrangendo desde atividades simples às mais complexas, direcionadas ao indivíduo e seus familiares ${ }^{(4)}$. O cuidado domiciliar pode ser operacionalizado por meio da visita e da internação domiciliar, e objetiva a realização de atividades de educação, prevenção, recuperação e manutenção da saúde das pessoas e de suas famílias ${ }^{(4)}$.

A expansão do cuidado domiciliar à saúde e sua associação com a ESF, como política pública de saúde emergente no Brasil, suscita a necessidade de se pensar em estratégias que representem um caminho para se seguir, unindo fatores organizacionais e ambientais e incluindo múltiplos fatores e componentes externos e internos, buscando a forma mais adequada de dispor os recursos existentes, além de direcionar o futuro ${ }^{(5)}$.

As estratégias para implementação da sistematização da assistência em saúde precisam ser construídas em coletividade, em especial no cuidado domiciliar, que é atividade complexa incorporada aos objetivos da ESF. Isso permite a atuação consciente dos profissionais envolvidos, otimizando os recursos existentes frente ao ambiente onde atuam.

Essas estratégias devem ser elaboradas não como simples sugestões desvinculadas da realidade existente, mas sim como forma de articular as singularidades do cuidado domiciliar, a intencionalidade de atuação da ESF e os recursos materiais e humanos disponíveis. Assim, este artigo tem o objetivo de explicitar estratégias que, vinculadas à realidade, operacionalizem avanços no cuidado domiciliar na ESF.

\section{METODOLOGIA}

Realizou-se um estudo intitulado "A prática de cuidado domiciliar dos profissionais do Programa Saúde da Família"(6), que utilizou a metodologia da pesquisa-ação, a qual procura trabalhar problemas pertinentes às diferentes práticas sociais, propondo transformar a situação investigada com estreita associação à teoria $^{(7)}$. Nela é valorizada a interação e a busca da autonomia coletiva, ao considerar a atuação conjunta de pesquisadores e atores envolvidos na situação $0^{(8)}$, para identificar problemas coletivos, buscar e experimentar soluções em situação real, com produção e uso de conhecimento ${ }^{(7)}$.

A pesquisa-ação caracteriza-se por ser críticointerrogativa, pois ao questionar sobre os problemas da situação atual dos atores envolvidos apresenta caráter interrogativo e, em contraponto, é crítica por não aceitar explicações de senso comum ${ }^{(8)}$.

Para este estudo, seguiu-se o caminho metodológico que compreende as seguintes etapas: fase exploratória, tema da pesquisa, colocação dos problemas, lugar da teoria, hipóteses, seminário, campo de observação, amostragem e representatividade qualitativa, coleta de dados, aprendizagem, saber formal e informal, plano de ação e divulgação dos resultados ${ }^{(7)}$. Essas, no entanto, não foram seguidas em uma ordem fixa, pois no desenvolvimento deste tipo de pesquisa o planejamento é muito flexível, havendo um constante vaivém entre cada etapa ${ }^{(7)}$.

O local do estudo foi uma unidade de saúde ESF de um município de grande porte, tendo como pesquisados-participantes todos os 27 profissionais de saúde desta unidade, incluindo enfermeiros, auxiliares de enfermagem, médicos, dentistas, auxiliares de consultório dentário, técnico de higiene dental e autoridade sanitária local.

O estudo foi realizado de julho de 2005 a julho 
de 2008 e aprovado pelo Comitê de Ética em Pesquisa do Setor de Ciências da Saúde da Universidade Federal do Paraná e pelo Comitê de Ética da Secretaria Municipal de Saúde do município de Curitiba (Folha de rosto SISNEP 070323; Protocolo de aprovação 136/2005).

A coleta de dados ocorreu por meio da observação assistemática participante e o registro dos dados foi realizado em diário de campo e por meio de notas teóricas, observacionais e metodológicas/operacionais, em diferentes momentos: no acompanhamento das 179 visitas domiciliares e em 6 seminários realizados após reuniões de equipe na unidade.

Este artigo representa uma parte dos resultados alcançados com o estudo e diz respeito às estratégias para operacionalização de avanços na prática do cuidado domiciliar desenvolvido na ESF. Ao trabalhar os problemas relacionados a essa prática, a pesquisaação viabilizou que os próprios sujeitos pesquisados apontassem, a partir de sua vivência profissional, estratégias para o aperfeiçoamento dessa modalidade de atenção à saúde.

\section{RESULTADOS E DISCUSSÃO}

As estratégias emergiram da problematização da prática de cuidado domiciliar na ESF, quando pesquisados e pesquisadores analisaram falhas e po-

Quadro 1 - Organização das estratégias em categorias tenciais soluções em sua prática profissional. A partir daí, compreenderam-se componentes estruturais, profissionais e institucionais que operacionalizam essa prática, tendo em vista a característica críticointerrogativa desse estudo. Os dados provenientes da observação participante e das notas em diário de campo, tanto nos seminários quanto nas visitas domiciliares, foram utilizados para a identificação de 19 estratégias (Quadro 1).

Ao analisar essas estratégias, perceberam-se aspectos em comum que versavam sobre: planejamento e organização do serviço; preparo profissional; condições que permeiam o cuidado domiciliar; revisão e fortalecimento dos saberes; melhor proveito da realidade e discussões integradoras. Identificados esses aspectos, os pesquisadores optaram por utilizá-los como títulos para a categorização das estratégias.

Esta categorização revelou um olhar críticoreflexivo dos profissionais de saúde, ao reconhecerem falhas e valorizarem pontos positivos em sua prática de cuidado domiciliar, propondo assim estratégias que representem avanços para a ESF.

O planejamento e organização do serviço são importantes para que se consiga evidenciar avanços em qualquer setor, principalmente na ESF, que representa uma inovação nas práticas de saúde no Brasil, para viabilizar a organização dos sistemas locais de saúde ${ }^{(9)}$.

\begin{tabular}{|ll|}
\hline CATEGORIAS & ESTRATÉGIAS \\
Planejamento e & - Estabelecimento de normas e rotinas \\
organização do serviço & - Elaboração de critérios para inclusão, exclusão e alta de usuários, para \\
& o direcionamento das visitas domiciliares e para elegibilidade do profissional \\
Preparo profissional & - Priorização de casos para o cuidado domiciliar \\
& - Educação permanente \\
& - Liderança para as práticas de cuidado domiciliar \\
& - Estabelecimento de vínculo entre profissional e comunidade atendida \\
& - Conhecimento de si do profissional \\
Condições que permeiam o & - Compreensão da importância da família \\
cuidado domiciliar & - Integração entre cuidado domiciliar e a ESF \\
& - Conhecimento dos profissionais acerca de sua área de abrangência \\
Revisão e fortalecimento & - Participação e co-responsabilização popular \\
dos saberes & - Reforço do conhecimento das atribuições profissionais \\
& - Aprofundamento teórico sobre produtividade, profissionalismo, ética, domicílio \\
Melhor proveito da realidade & e educação em saúde \\
& - Atualização sobre as políticas públicas de saúde \\
Discussões integradoras & - Sensibilização dos profissionais quanto ao significado de mudanças \\
& - Relevância das facilidades existentes no cuidado domiciliar \\
& - Busca do entendimento de conceito e objetivos do cuidado domiciliar \\
& - Reunião multiprofissional \\
& - Discussão de casos pelos profissionais após a visita domiciliar \\
\hline
\end{tabular}


A falta de padronização das ações profissionais foi citada no estudo como causadora de um trabalho não condizente com os princípios de equidade e igualdade no atendimento à saúde do Sistema Único de Saúde (SUS) no atendimento à saúde. Com isso, identificou-se como estratégia o estabelecimento de normas e rotinas que representam uma burocratização necessária devido à complexidade de ações envolvidas nos serviços de saúde. Os profissionais demonstraram que atuam e se organizam melhor quando há rotinas e protocolos que refletem sobre melhorias em suas práticas, se aliados à vigilância sanitária.

Outra questão foi que os participantes da pesquisa referiram dificuldades em estabelecer critérios de inclusão, exclusão e alta de usuários, e constatou-se a realização de atendimentos domiciliares desnecessários e o não-desligamento de famílias que poderiam promover seu processo de saúde buscando auxílio na própria unidade de saúde. Entretanto, a determinação destes critérios poderia facilitar o planejamento do cuidado domiciliar e melhorar sua frequência e regularidade, bem como a utilização dos recursos disponíveis. Além disso, os critérios para direcionamento das visitas podem ser facilitadores da prática profissional porque viabilizam melhor aproveitamento do percurso e do fluxo das mesmas.

A elegibilidade do profissional adequado também se destaca, pois ela pode evitar que os profissionais de saúde despendam seu tempo em atendimentos que não precisam de sua presença; contudo, verificou-se nesta pesquisa a dificuldade, por parte dos trabalhadores, em designar o profissional adequado para realizar o cuidado domiciliar.

O estabelecimento desses critérios, conforme apontado pelos sujeitos pesquisados, é essencial para que se possa ter a priorização de casos para o cuidado domiciliar, ponderando que a ESF propõe atendimentos voltados a domicílios onde há risco para adoecimento e onde já existam problemas de saúde instalados. O estudo aponta que por causa da não priorização de casos, algumas famílias que não necessitariam do cuidado domiciliar acabam recebendo esta assistência, ou por uma postura profissional paternalista e protetora, ou por uma postura de comodidade das pessoas que se isentam da responsabilidade sobre sua saúde. Dessa forma, não há a otimização dos recursos disponibilizados para o cuidado domiciliar.

Para que os profissionais de saúde possam desenvolver uma boa prática em saúde, o preparo profissional é uma questão relevante, principalmente ao considerar o cuidado domiciliar dentro da ESF, que almeja nova compreensão do processo saúde-doença e engloba, além do indivíduo, a sociedade, a família, a cultura, a política, entre outros fatores.

A educação permanente pode servir como instrumento de capacitação para que os profissionais de saúde aprimorem ou adquiram conhecimentos e habilidades, e desenvolvam postura crítica, autoavaliação, autoformação e autogestão ${ }^{(10)}$. Ela é importante no cuidado domiciliar, visto que a equipe de saúde nem sempre é a mesma, o que exige dos profissionais preparo e comprometimento permanentes.

Além disso, utilizar alguns instrumentos gerenciais torna o profissional mais capacitado para atuar no cuidado domiciliar, sendo um deles a liderança, descrita como um processo de influenciar ou conduzir um grupo numa determinada direção para o alcance de objetivos $^{(11)}$. Os profissionais desta pesquisa apontaram a necessidade de um líder para coordenar a equipe, a fim de direcionar a necessidade de atenção às atividades profissionais e ressaltaram que, para a efetividade de uma liderança, deve haver respeito pelas opiniões diversas e direcionamento para discussões e reflexões pertinentes à prática profissional da equipe.

Outra questão importante é o conhecimento de si do profissional, para identificar suas facilidades, dificuldades ou limitações e assim refletir e encontrar meios de superar seus próprios obstáculos, aperfeiçoar sua prática e compartilhar suas habilidades com a equipe da ESF. Ter consciência dos seus próprios sentimentos, atitudes e ações possibilita ao profissional encontrar meios eficientes de ajudar os outros.

O cuidado domiciliar na ESF não se dá de forma aleatória, mas reúne condições que permeiam sua realização, conforme citadas pelos sujeitos pesquisados: compreensão da importância da família; integração entre cuidado domiciliar e a ESF; conhecimento dos profissionais acerca de sua área de abrangência; participação e corresponsabilização popular.

Para entender a ESF, precisa-se compreender, em primeiro lugar, a família como promotora de saúde, como eixo estruturante da vida e unidade de cuidados das pessoas, ou ainda como uma entidade inserida numa realidade socioeconômica. Para os profissionais da ESF há a dificuldade de ampliação do atendimento para toda a família, pois ainda o foco é no indivíduo doente. Isso permite refletir que a compreensão da importância da família no cuidado domiciliar possibilita aos profissionais uma atuação mais ampla e eficaz, já que o cuidador familiar é quem 
está mais próximo do usuário atendido ${ }^{(12)}$.

A integração entre cuidado domiciliar e ESF está, principalmente, relacionada ao fato de que ambos mediam a aproximação dos profissionais com a sociedade onde os indivíduos estão inseridos e, dessa forma, possibilitam que a atenção em saúde envolva a promoção, prevenção e recuperação da saúde. Os profissionais indicaram que isso facilita a compreensão da dimensão social na qual estão inseridas as famílias, possuindo características peculiares e também comuns. No cuidado domiciliar, essa aproximação ocorre na residência, onde acontece um processo de interação entre o profissional de saúde e o usuário/família ${ }^{(13)}$.

Também se compreende que essa integração entre cuidado domiciliar e ESF é permeada pela necessidade de conhecimento da área de abrangência ${ }^{(8)}$. A definição do território de atuação é uma responsabilidade das equipes de atenção básica, e na unidade de saúde ESF, o conhecimento acerca desta área se torna ainda mais relevante. Quando isso não ocorre, há um prejuízo à população assistida, pois a falta de conhecimento dos profissionais inviabiliza o estabelecimento das necessidades prioritárias de saúde dessa população $0^{(12)}$.

No entanto, não basta conhecer a área de abrangência, mas cabe ao profissional atuar de forma a promover a participação popular, corroborando os princípios do SUS, pois é fundamental a atuação da população na sua condição de vida e saúde, sendo que o trabalhador de saúde pode, por meio de sua prática profissional, buscar promover a emancipação dos sujeitos da comunidade onde atua.

Além disso, diante da prática de cuidado domiciliar, os profissionais pesquisados apontaram que a revisão e fortalecimento dos saberes servem como ferramentas para relembrar e melhor compreender determinados assuntos de seu cotidiano, além de permitir a resolução de problemas e o esclarecimento de dúvidas, o que facilita a organização e execução do cuidado.

A necessidade de reforço do conhecimento das atribuições profissionais se dá diante da dificuldade encontrada pelos sujeitos na padronização e coordenação das ações profissionais. Com isso, espera-se que cada profissional da equipe da ESF conheça detalhadamente e compreenda suas atribuições, tendo em mente qual o tipo de atividade pode desenvolver, pois o estabelecimento destas atribuições deve minimizar a confusão de papéis, o aparecimento de conflitos e a utilização inadequada dos recursos humanos disponíveis, otimizando o cuidado domiciliar.

O aprofundamento teórico sobre produtividade, profissionalismo, ética, domicílio e educação em saúde surge como estratégia mediante as dificuldades citadas pelos profissionais, por acreditar que o conhecimento pode oportunizar a melhoria de atuação nesses aspectos.

A exigência de produtividade de ações em saúde foi citada pelos sujeitos da pesquisa, demonstrando maior preocupação institucional em atingir metas do que em manter a qualidade dos serviços prestados. É importante que o trabalhador mantenha o profissionalismo e princípios éticos, a fim de evitar que a produtividade se resuma a números, consultas e exames.

Além disso, o profissional envolvido no cuidado domiciliar deve considerar a dinamicidade do domicílio e o espaço relacional das famílias, para entender o domicílio como um local adequado à realização de cuidados, tais como ações de educação em saúde $^{(8)}$. A educação em saúde é uma ação planejada, que busca criar condições para produzir mudanças comportamentais desejáveis em relação à saúde ${ }^{(14)}$. Ela evidencia-se como ponto-chave na prática do cuidado domiciliar, pois permite resolver problemas potenciais com antecedência.

Contudo, observou-se que a educação em saúde, na prática do cuidado domiciliar, acontece de maneira falha, superficial, descontextualizada e divergente das necessidades do paciente e da família. Sobre isso, algumas razões apontadas pelos profissionais foram: tempo curto do atendimento domiciliar; falta de vínculo com a família e de postura profissional. Com relação a este último fator, destaca-se a relevância das orientações serem dirigidas às famílias de uma forma não impositiva, valorizando a experiência e troca de saberes.

Os profissionais ainda relataram a necessidade de atualização no que tange às políticas públicas de saúde, de modo a realizar atividades convergentes com as atuais práticas de saúde e educação popular. Nesse sentido, há importância em conhecer melhor as Políticas Públicas de Saúde, que devem ser destinadas e pertinentes à realidade do local em que se aplicam.

Dessa forma, a adaptação do profissional às transformações propostas pela ESF é uma questão fundamental para que se possa ter um melhor proveito da realidade para o cuidado domiciliar. As mudanças representam formas de adaptar-se às exigências do mercado de trabalho e, no caso do cuidado domiciliar na ESF, possibilitam o real alcance das propostas de 
saúde pública existentes; contudo, como relataram os sujeitos pesquisados, mudanças por razões não significativas podem ser geradoras de conflitos e tensões.

Observou-se a necessidade constante de motivação da equipe estudada no que tange as discussões da prática em relação a modificações, visto que os profissionais se apresentam resistentes às mudanças, pois estas exigem, de imediato, maior dedicação, tempo e disposição. Assim, percebe-se que não basta apontar, mas sim estimular a sensibilização dos profissionais quanto ao significado de mudança ${ }^{(11)}$.

Embora mudanças sejam importantes, as facilidades já existentes também devem ser consideradas, visto que representam pontos favoráveis para a efetividade da atuação profissional ${ }^{(12)}$. Os sujeitos demonstraram dificuldade em pontuar quais são as suas facilidades no cuidado domiciliar e em visualizálas como avanços conquistados. Por isso, aponta-se a necessidade de valorização das facilidades e do potencial de melhorias, compreendendo a realidade existente em cada situação vivenciada e a interdependência das variáveis envolvidas ${ }^{(12)}$.

Por fim, para a melhor atuação no cuidado domiciliar, destaca-se a necessidade de realização de discussões integradoras entre os profissionais, como forma de avaliar seus conhecimentos e sua prática profissional, e determinar se o cuidado prestado pela equipe está atendendo efetivamente as necessidades do usuário e sua família.

No início do estudo, os sujeitos demonstraram interesse em discutir e refletir sobre o cuidado domiciliar e relataram desconhecimento sobre as categorias relativas a esse tipo de atenção em saúde, apesar de trabalharem com ela. A maior necessidade de conhecimento foi em relação à visita domiciliar. Assim sendo, a busca pelo entendimento do conceito e dos objetivos do cuidado domiciliar representa uma importante estratégia para ampliar a objetividade de cada ação realizada no domicílio. Vale ainda salientar que esse conhecimento é indispensável para que cada profissional da ESF possa planejar, coordenar, organizar e dirigir suas ações, além de discutir esses aspectos com a equipe, sempre que possível.

Uma das principais dificuldades para o trabalho em equipe citadas foi a falta de integração no trabalho, visto que, embora o cuidado domiciliar seja uma tentativa de reversão do modelo de atenção à saúde, continua reproduzindo o trabalho centrado no médico. Assim, identificou-se a necessidade de integração profissional na prática, com trocas de informação, otimização de tempo e recursos humanos, o que pode ser viabilizado por meio de reunião multiprofissional e discussão de casos após a visita domiciliar.

O debate de casos após a visita domiciliar ocorre a fim de reconhecer as particularidades e reais necessidades de saúde da população, visualizando não só o usuário ou sua doença, mas o todo envolvido, para que com isso, e ainda com a visão de diferentes profissionais, a equipe possa elaborar um plano de intervenção que englobe todos os aspectos.

\section{CONSIDERAÇÕES FINAIS}

A ESF faz parte da política pública brasileira que aponta novos horizontes para a saúde no país, entrementes, as modificações propostas ainda não se encontram consolidadas. É preciso compreender as dificuldades de implantação da ESF e, principalmente, reconhecer as possibilidades e avanços que surgem nesse processo.

Assim, embora se perceba que ainda há muito a fazer, já é possível identificar melhorias e estas devem ser consideradas. Nesta pesquisa, observou-se que os profissionais de saúde reconheceram a necessidade de implementação de estratégias organizadoras da prática de cuidado domiciliar, a fim de operacionalizar avanços reais e efetivos nesta área.

As estratégias elencadas servem como norte, pois emergiram de lacunas existentes na prática profissional do cuidado domiciliar na ESF e, desta forma, podem auxiliar na consolidação desta incipiente prática de saúde. Estas estão direcionadas aos profissionais e à população assistida, sem com isso eximir o Estado de suas funções nesse contexto.

Contudo, necessita-se do envolvimento de todos os níveis políticos - participação popular, profissional e estatal - para que aconteça, pela fusão desses agentes, a modificação da realidade de saúde do Brasil e, assim, a proposta de atenção básica de saúde seja efetivamente alcançada. Além disso, acredita-se que incitar a atuação crítico-reflexiva dos profissionais de saúde pode contribuir para a elaboração de estratégias que cada vez mais operacionalizem a prática qualificada em saúde.

\section{REFERÊNCIAS}

1. Ministério da Saúde (BR). Política Nacional de Atenção Básica. 2007. [acesso em 01 dez 2009]. Disponível: http://tiny.cc/sg9rj 
2. Fernandes LCL, Bertoldi AD, Barros AJD. Utilização dos serviços de saúde pela população coberta pela Estratégia de Saúde da Família. Rev Saúde Pública. [Internet] 2009;43(4) [acesso em 17 set 2009]. Disponível: http://tiny.cc/lte0k

3. Giacomozzi CM, Lacerda MR. A prática da assistência domiciliar dos profissionais da estratégia de saúde da família. Texto \& Contexto Enferm. 2006;15(4): 645-53.

4. Lacerda MR, Giacomozzi CM, Oliniski SR, Truppel TC. Atenção à saúde no domicílio: modalidades que fundamentam sua prática. Saúde Soc. 2006;15(2):88-95.

5. Chiavenato I. Administração: teoria, processo e prática. São Paulo:Makron Books; 2000.

6. Lacerda MR. A prática de cuidado domiciliar dos profissionais do Programa Saúde da Família. Curitiba [Projeto Programa de Iniciação Científica - CNPq]. Curitiba (PR): Universidade Federal do Paraná; 2005.

7. Thiollent M. Metodologia da pesquisa-ação. São Paulo: Cortez; 2003.

8. Giacomozzi CM. As práticas de cuidado domiciliar à saúde desenvolvidas pelos profissionais da ESF [dissertação]. Curitiba (PR): Universidade Federal do Paraná; 2007.

9. Viana ALD, Dal Poz MR. A reforma do sistema de saúde no Brasil e o Programa de Saúde da Família. Physys. 2005;15 Suppl:225-64.

10. Oliveira MAN. Educação a distância como estratégia para a educação permanente em saúde: possibilidades e desafios. Rev Bras Enferm. 2007;60(5):585-9.

11. Marquis BL, Huston CJ. Administração e liderança em enfermagem: teoria e prática. Porto Alegre: Artmed; 2005.

12. Gomes IM. Os profissionais de saúde da família e sua prática em cuidado domiciliar [relatório técnico]. Curitiba (PR):Universidade Federal do Paraná; 2008.

13. Lacerda MR, Zagonel IPS, Martins SK. Standards of the nursing knowledge and its interface to the attendance to the domiciliary - a literature review. Online Braz J Nurs. [Internet] 2006; 5(2) [acesso em 8 dez 2009]. Disponível: http://tiny.cc/tenjb

14. Santos AS. Health education: reflection and applicability in primary health care. Online Braz J
Nurs. [Internet] 2006; 5(2) [acesso em 2 dez 2009]. Disponível: http://tiny.cc/b6u0h. 